Meta

Journal des traducteurs

Translators' Journal

\title{
La neurophysiologie du bilinguisme
}

\section{Yvan Lebrun}

Volume 29, numéro 1, mars 1984

Cerveau, langage et traduction

URI : https://id.erudit.org/iderudit/003432ar

DOI : https://doi.org/10.7202/003432ar

Aller au sommaire du numéro

Éditeur(s)

Les Presses de l'Université de Montréal

ISSN

0026-0452 (imprimé)

1492-1421 (numérique)

Découvrir la revue

Citer cet article

Lebrun, Y. (1984). La neurophysiologie du bilinguisme. Meta, 29(1), 36-43.

https://doi.org/10.7202/003432ar d'utilisation que vous pouvez consulter en ligne.

https://apropos.erudit.org/fr/usagers/politique-dutilisation/ 


\section{LA NEUROPHYSIOLOGIE DU BILINGUISME}

YVAN LEBRUN

Il y a une vingtaine d'années, la très vénérable revue de neurologie Brain publia un article qui, à la différence de ceux qu'elle offrait généralement à ses lecteurs, ne traitait pas de problèmes neurologiques. Il y était au contraire question d'apprentissage des langues. L'auteur n'en appartenait pas moins à la profession médicale. Il en était même un très distingué représentant. Neurochirurgien réputé, il avait été professeur à l'Université McGill de Montréal et le premier directeur du Montreal Neurological Institute. D'où lui venait donc cet intérêt pour l'apprentissage des langues? Avant d'entreprendre ses études de médecine, l'auteur de l'article, Wilder Penfield (1965), avait été, pendant un an, professeur d'allemand dans une école pour garçons. On pourrait penser que, malgré une glorieuse carrière en médecine, il avait gardé la nostalgie de cette expérience d'enseignant. En fait, tel ne paraît pas avoir été le cas. Penfield considérait qu'il avait été un piètre professeur de langues. Ce sont bien plutôt ses fonctions de neurochirurgien qui l'amenèrent à s'intéresser aux mécanismes cérébraux de l'acquisition du langage et en particulier à la manière dont un cerveau apprend et utilise plusieurs langues. À première vue, il peut paraîtrre surprenant que ce soit par le biais de la pathologie cérébrale qu'ait été abordée la neurophysiologie du bilinguisme. À la réflexion, il apparaît toutefois qu'il n'existe guère d'alternative. En effet, celui qui s'intéresse au fonctionnement des organes périphériques de la parole peut approcher directement l'objet de son étude. Par la vue, l'audition, le toucher et l'introspection, il peut découvrir de nombreux aspects de la physiologie de la parole. Et pour l'examen des parties de l'appareil phonatoire qui sont cachées au regard, il peut recourir à des techniques comme la radiographie, la cinéradiographie, la palatographie, l'électromyographie, la stroboscopie, ou l'électroglottographie. Toute différente est la situation du chercheur qui souhaite découvrir quelles structures cérébrales président à l'expression orale et comment ces structures fonctionnent. Le système nerveux central, lui, ne se laisse pas observer directement, et les techniques instrumentales dont on dispose actuellement pour en étudier l'activité dans l'acte de parole ne permettent que des observations très grossières. Qu'il s'agisse d'investigations électroencéphalographiques, de mesures du débit sanguin cérébral ou d'enregistrements de potentiels évoqués, les données ainsi recueillies ne renseignent que vaguement sur la neurophysiologie de la parole. Force est donc au chercheur de recourir à des procédés indirects pour tenter de comprendre comment le cerveau dirige l'activité verbale. En gros, ces procédés sont de deux types. Le premier est de nature expérimentale : en soumettant des sujets à une tâche donnée et en étudiant la façon dont ils s'acquittent de cette tâche, on s'efforce d'approcher un aspect déterminé du fonctionnement cérébral. Cette technique, très largement utilisée par la psychologie expérimentale, soulève fréquemment plus de problèmes qu'elle n'en résout. En effet, d'une expérience à l'autre, les résultats souvent varient, sans doute parce qu'interviennent dans l'exécution de la tâche proposée des facteurs qui échappent au contrôle de l'expérimen- 
tateur ou que celui-ci ne soupçonne même pas. Si bien qu'en dépit du zèle mis par les chercheurs à inventer des tâches susceptibles de nous éclairer sur le comportement du cerveau dans les activités verbales, peu de conclusions définitives ont pu être tirées jusqu'ici des nombreuses expériences réalisées.

L'autre abord indirect du fonctionnement cérébral est clinique. Ici, il s'agit d'essayer de comprendre la physiologie du système nerveux central à travers les désordres fonctionnels qu'entraînent les lésions de ce système. Appliquée au bilinguisme, cette approche implique que l'on étudie les troubles du langage pouvant apparaître chez des sujets qui connaissent deux ou plusieurs langues et qui, a un moment donné, sont victimes d'une atteinte cérébrale. Que nous apprend cette neurologie du bilinguisme? L'observation peut-être la plus curieuse est que la pathologie cérébrale n'affecte pas toujours de façon uniforme les différentes langues que connaît le malade. Ainsi, le patient peut perdre temporairement ou définitivement la possibilité d'utiliser une langue alors qu'il conserve - au moins partiellement - la possibilité d'en employer une autre. Les Gloning (1965) ont rapporté le cas d'une femme dont la langue maternelle était le slovène, mais qui connaissait l'allemand depuis son enfance. À l'âge de 28 ans, elle émigra en Argentine. Elle vécut dix-huit ans à Buenos-Aires et y apprit à parler très couramment l'espagnol. Elle revint alors en Europe et ne se servit plus que du slovène et de l'allemand. Elle garda toutefois une profonde nostalgie de l'Amérique du Sud. À 57 ans, elle développa une tumeur cérébrale frontale gauche, qui entraîne une aphasie. La malade continuait de comprendre le slovène et l'espagnol, mais éprouvait des difficultés à comprendre l'allemand. Elle ne parlait pas spontanément. Si on l'interrogeait en slovène ou en espagnol, elle répondait en espagnol ou bougeait les lèvres sans produire de sons. La dénomination d'objets était meilleure en espagnol que dans sa langue maternelle et était très difficile en allemand. La malade pouvait encore lire dans les trois langues mais ne pouvait plus s'exprimer par écrit dans aucune. Après un certain temps, l'aphasie s'aggrava : la patiente ne fut bientôt plus capable que d'utiliser quelques mots espagnols. Elle mourut un peu plus tard.

Ce cas montre non seulement qu'il peut y avoir atteinte différentielle des langues que connaît l'aphasique, mais aussi que la langue la moins affectée n'est pas nécessairement la langue maternelle. Il peut même arriver que seule l'utilisation de la langue maternelle soit rendue difficile par la lésion cérébrale. Paradis (1981) a rapporté le cas d'un patient malgache d'origine indienne. Né à Madagascar dans une famille qui parlait le gujarati, ce patient avait appris très jeune le malgache en plus de sa langue maternelle. Toute sa scolarité se fit en français. Avec les membres de sa famille et avec ses amis indiens de Madagascar, le malade parlait gujarati. Il utilisait le malgache dans ses rapports avec la population locale. Devenu comptable dans l'entreprise de son père, il employait surtout le français pour son travail. Vers l'âge de 26 ans, le patient commença à présenter des crises d'épilepsie de type Bravais-Jackson dans le bras gauche et une diminution de la force musculaire dans la jambe gauche. Un examen tomodensitométrique révéla une zone hyperdense dans la partie médiane de la circonvolution prérolandique droite. Le malade fut trépané et un kyste d'origine parasitaire fut trouvé dans cette circonvolution. On pratiqua l'exérèse du kyste. Après l'intervention, le malade présenta une hémiparésie gauche avec un déficit verbal objectivable seulement en gujarati. Dans cette langue, il éprouvait des difficultés à commencer ses phrases, comme si un spasme l'empêchait de parler. Des blocages de ce genre se produisaient aussi en cours de phrase. Le patient expliquait - en français - qu'il savait quels mots utiliser mais avait du mal à " les faire sortir ". Il devait souvent s'y reprendre à plusieurs fois pour dire le mot désiré. Ses efforts rappelaient ce que l'on observe parfois chez le bègue. La répétition de logatomes était aussi perturbée. Il existait en outre un déficit véritablement aphasique : le 
malade comprenait mal certaines consignes de la version nouvelle du Token Test (objets au lieu de figures géométriques), éprouvait des difficultés d'évocation du mot et sa fluence verbale était diminuée. Curieusement, aucun de ces troubles ne fut observé en français ou en malgache. En fait, le patient ne présentait dans ces deux langues aucun déficit cliniquement décelable. Seule la langue maternelle était affectée.

Après lésion cérébrale, certains polyglottes présentent non pas une atteinte différentielle mais une récupération différentielle de leurs langues. Bychowski (1919) a décrit un patient dont la langue maternelle était le polonais. Comme il habitait près de la frontière germano-polonaise, cet homme avait aussi appris l'allemand. À l'âge de 18 ans, il passa d'ailleurs huit mois en Allemagne. Il comprenait également le russe. Devenu militaire, il fut envoyé en Russie où il apprit à parler, lire et écrire la langue russe. Ses supérieurs étaient tous russes, mais, avec ses compagnons de chambre, le jeune homme continuait de parler polonais. En 1915, une blessure par balle endommagea son hémisphère cérébral gauche. Hémiplégique et complètement aphasique, le soldat fut transporté dans un hôpital de Moscou. Dix mois après l'accident, la compréhension verbale commença à lui revenir : il put comprendre ce que lui disait une infirmière russe qui l'avait pris en affection. Aidé par cette infirmière, le malade récupéra une partie de sa capacité à s'exprimer en russe. Deux ans et demi après le début de l'aphasie, le patient pouvait à nouveau comprendre et répéter des phrases en polonais, mais il éprouvait d'énormes difficultés à parler, lire ou écrire cette langue. Il lui était beaucoup plus aisé de parler le russe, sans toutefois que son débit verbal fût redevenu fluide dans cette langue. Le malade restait incapable d'utiliser l'allemand, sous quelque forme que ce fût.

Ces cas cliniques montrent que, contrairement à ce que pensait Ribot (1881), les connaissances linguistiques les plus précocement acquises ne sont pas toujours celles qui résistent le mieux, ou sont le mieux recouvrées, après lésion cérébrale. Ni la précocité des acquisitions verbales ni la fréquence de leur utilisation ne sont une garantie de meilleure préservation en cas d'atteinte du système nerveux central. En fait, même des activités verbales quotidiennement répétées, de façon plus ou moins stéréotypée, peuvent être plus perturbées que des activités verbales plus complexes et plus élaborées. De Menasce (1973), qui était prêtre, fut, à 57 ans, victime d'un accident vasculaire cérébral qui rendit difficile l'utilisation de l'allemand, alors que l'anglais, sa langue maternelle, n'était que peu affecté. Mais c'est le latin qui souffrit le plus : dans les semaines qui suivirent l'agression cérébrale, le malade fut totalement incapable de réciter par coeur, dans cette langue, le Pater, l'Ave et le Canon de la messe, alors qu'il avait récité ces prières chaque jour pendant de très nombreuses années. De même, une religieuse observée par Paradis et al. (1982) et qui fut victime d'un traumatisme crânien, retrouva, après un certain temps, la possibilité de s'exprimer en français et en arabe, alors qu'elle restait incapable de réciter par coeur l'Ave Maria en latin ; elle avait pourtant dit cette prière plusieurs fois par jour pendant les quinze années qui avaient précédé le traumatisme.

Parfois, le polyglotte cérébro-lésé ne conserve, ou ne retrouve, que la possibilité d'utiliser une seule des langues qu'il connaissait. Pitres (1895) a rapporté le cas d'un homme qui, né en Lorraine, avait appris, dès l'enfance, le français et l'allemand. "Il fit, plus tard, de sérieuses études classiques de grec et de latin. Marié très jeune à une Basquaise, il se fixa à Tarbes à l'âge de vingt-deux ans, et y apprit le basque, qu'il arriva bientôt à parler et à prononcer correctement. Plus tard, il apprit ... l'anglais, l'espagnol et l'italien. " $\grave{A}$ cinquante-six ans, ce polyglotte fut victime d'un accident cérébrovasculaire qui ne lui laissa que la langue française ; il était incapable de comprendre encore ou de parler les autres langues qu'il avait apprises. On voit donc qu'une lésion cérébrale peut ramener un polyglotte à la condition d'unilingue. Quant aux unilingues, il arrive parfois que la pathologie cérébrale leur donne l'apparence d'étrangers. Monrad- 
Krohn (1947) a eu l'occasion d'examiner une Norvégienne qui ne connaissait que sa langue maternelle mais qui, à la suite d'un traumatisme crânien, se mit à la parler avec des intonations et des accentuations si curieuses que les Norvégiens qui ne la connaissaient pas la prenaient pour une Allemande. Quant à Alajouanine (1968:69), il signale avoir observé, à la Salpêtrière, un certain nombre de malades " qui parlaient avec un accent rappelant plus ou moins fidèlement l'accent anglais ou l'accent allemand, et même quelquefois un accent belge ". Dans son livre sur les troubles de la voix, Aronson (1980: 119) mentionne que, dans les archives médicales de la Mayo Clinic, il a retrouvé 13 cas d'accent étranger après lésion cérébrale. Douze autres cas lui sont connus par la littérature. Parmi ces 25 patients, il y avait 16 femmes et 9 hommes. Cette différence entre les sexes est-elle due à l'échantillonnage, c'est-à-dire qu'elle résulte du pur hasard et qu'il n'y a pas lieu de s'y attarder ? Ou les déviances intonatives et accentuelles sont-elles réellement plus fréquentes chez les femmes au même titre que la dysphonie s'observe plus souvent chez des sujets féminins? Une troisième possibilité est que la dysprosodie se manifeste avec la même fréquence dans les deux sexes, mais qu'elle frappe plus chez la femme que chez l'homme. Le peu de données dont on dispose à l'heure actuelle ne permet pas de choisir entre ces trois hypothèses.

Il est intéressant de noter que les accents que l'on croyait reconnaître chez les 25 malades inventoriés par Aronson étaient des plus divers : alsacien, polonais, allemand, gallois, suédois, norvégien, espagnol, écossais, germanique, slave ... Cette diversité donne à penser qu'il s'agit en fait dans la plupart, sinon dans la totalité des cas, de pseudo-accent : à la suite de son affection cérébrale, le malade éprouve des difficultés à articuler correctement et à produire les schèmes intonatifs typiques de sa langue maternelle. L'observateur est frappé par la façon anormale dont parle le patient, et rapproche ce qu'il entend de ce qu'il a entendu - ou cru entendre - lorsque parlaient des étrangers. Mais il s'agit là d'impressions subjectives et il est probable que l'on constaterait de notables différences si l'on comparait, sur la base d'analyses phonétiques sérieuses, la production orale du malade et celle d'étrangers auxquels il est censé ressembler.

Mais revenons aux polyglottes. Les cas cliniques cités plus haut montrent que les diverses langues que connaît un individu plurilingue peuvent ne pas être toutes affectées avec la même intensité par un accident cérébral. À ces différences quantitatives s'ajoutet-il parfois des différences qualitatives? En d'autres termes, peut-il arriver que les difficultés éprouvées par le polyglotte après atteinte du système nerveux central varient selon les langues non seulement en importance, mais aussi en nature? Existe-t-il des plurilingues qui présentent une forme d'aphasie dans une langue et une autre forme dans une autre langue? La possiblité de deux aphasies différentes chez un même sujet a été évoquée par plusieurs auteurs, mais les observations cliniques font encore défaut qui montreraient clairement que ce type de dissociation peut exister et qu'un malade peut, par exemple, comprendre bien une langue mais avoir de la difficulté à la parler (aphasie motrice), tandis qu'il comprend mal une autre langue et la parle de façon fluide mais aberrante (aphasie sensorielle).

Si la possibilité d'une atteinte qualitativement différente des diverses langues que connaît le polyglotte reste à démontrer, l'existence de déficits quantitativement différents est un fait acquis. Les cas cliniques cités plus haut en sont la preuve. On peut dès lors s'interroger sur l'origine de ces atteintes différentielles. Lorsqu'une langue est mieux préservée ou mieux récupérée qu'une autre, est-ce parce que les deux langues ne dépendent pas exactement des mêmes circuits neuronaux et que la lésion a moins touché les circuits qui sous-tendent l'une que les circuits qui sous-tendent l'autre ? Cette question présuppose qu'il puisse y avoir des différences typographiques dans la représentation cérébrale des diverses langues que maîtrise le polyglotte. A-t-on des raisons de pen- 
ser qu'il en est peut-être ainsi ? Se pourrait-il que la zone corticale du langage ne soit pas homogène chez les individus plurilingues, ou tout au moins chez certains d'entre eux, et que des parties de cette zone soient affectées plus spécialement, ou exclusivement, à l'usage d'une seulement des langues connues? Ojemann et Whitaker (1978) et plus récemment Rapport, Tan et Whitaker (1978) ont cherché à répondre à cette question en appliquant, chez quelques patients polyglottes, la technique de la stimulation électrique d'un point du cortex pendant une épreuve de dénomination. Cette expérience se pratique lors d'une craniotomie sous anesthésie locale. Une partie du cortex étant découverte, généralement dans la partie gauche du cerveau, on stimule électriquement, à certains moments du test de dénomination, divers endroits de la matière grise exposée. Ces endroits sont stimulés tour à tour et pour chacune des deux langues que l'on veut comparer entre elles. L'expérience montre qu'à certains endroits du cortex, la stimulation électrique perturbe la dénomination également dans les deux langues. À d'autres, la perturbation est plus importante dans une langue que dans l'autre. À d'autres enfin, la stimulation électrique ne perturbe pas la dénomination. Les quatre chercheurs qui ont appliqué cette technique considèrent que là où le nombre d'erreurs de dénomination dans une langue est significativement plus élevé lors de la stimulation électrique que lors de l'absence de stimulation, on se trouve à un endroit du cortex qui est impliqué dans l'utilisation de cette langue. Les auteurs identifient ainsi un certain nombre de sites qui paraissent sous-tendre également les deux langues, et quelques sites où un nombre significatif d'erreurs sous stimulation est relevé pour seulement une des deux langues considérées. La découverte de tels points les conduit à penser que peut-être le substrat organique n'est pas exactement le même pour les deux langues, mais qu'il existe, au contraire, une localisation corticale quelque peu différente pour chacune d'elles. Une telle hypothèse est évidemment plausible. Elle a d'ailleurs déjà été proposée dans le passé sur des bases exclusivement cliniques. En effet, chez certains polyglottes présentant une atteinte différentielle de leurs langues, on peut se demander si la clause n'en est pas que les langues n'ont pas été ni apprises ni utilisées de la même manière, ce qui aurait eu pour effet de leur donner une représentation corticale différente au moins par certains côtés. Hinshelwood (1902) a décrit le cas d'un Anglais qui avait une fort bonne connaissance de trois langues étrangères : le français, le latin et le grec. À l'âge de 34 ans, cet homme eut une attaque qui entraîne une aphasie mixte. La situation s'améliora assez rapidement et il n'y eut bientôt plus que des difficultés de lecture. Le patient avait beaucoup de mal à lire l'anglais et souvent devait déchiffrer les lettres une à une pour arriver à identifier le mot. Curieusement, il n'éprouvait guère de difficulté à lire le grec. La lecture en français et en latin, tout en étant moins aisée qu'en grec, était nettement plus facile qu'en anglais. Après un certain temps, le malade put à nouveau lire dans les quatre langues, mais la lecture de l'anglais continua de lui demander plus d'effort de concentration que celle du latin ou du grec. Comment expliquer que la lecture de la langue maternelle fût plus affectée que celle de langues étrangères ? On peut penser que chez ce malade, l'anglais était, au niveau cérébral, organisé différemment du latin et du grec parce que les modalités d'acquisition de la langue maternelle avaient été différentes de celles des deux langues classiques. L'anglais oral avait été appris pendant la prime jeunesse et l'anglais écrit au début de la scolarité. Les deux langues classiques avaient été acquises plus tard et n'avaient jamais été parlées. Ces différences pourraient expliquer une localisation cérébrale non identique et, partant, une atteinte différentielle.

Dans certains cas, c'est la nature même de chacun des codes qui paraît être à l'origine d'une organisation différente des structures cérébrales impliquées. Sinon, comment expliquer que certains malades qui ont perdu la possibilité de comprendre la langue écrite, continuent de pouvoir utiliser les chiffres et de pouvoir lire les formules mathé- 
matiques (voir, par exemple, le cas rapporté par Ajax en 1967) ? De même, on a pu constater que chez certains sourds congénitaux qui ont appris à la fois le langage écrit traditionnel et la dactylographie, une lésion cérébrale peut entraver la production de l'un seulement de ces codes, bien qu'ils soient tous les deux produits manuellement et reposent l'un et l'autre sur le même système orthographique (Lebrun et Leleux 1981). Il est donc fort vraisemblable que le substrat organique varie, au moins dans certaines limites, avec la nature du code qu'il sous-tend.

Comme on le voit, l'étude de l'aphasie permet de soulever quelque peu le voile qui recouvre le fonctionnement du système nerveux central dans les activités verbales et de formuler quelques hypothèses sur la neurophysiologie du langage en général et du bilinguisme en particulier.

L'aphasie n'est d'ailleurs pas le seul trouble dont l'étude permette des conjectures sur l'organisation cérébrale du bilingue. Le bégaiement offre lui aussi un certain accès aux mécanismes compliqués qui assurent la maîtrise des langues. Ainsi, il a été observé à mainte reprise que le bègue cesse d'achopper lorsqu'il utilise une langue étrangère qu'il ne possède pas parfaitement. Aussi Itard recommandait-il que l'on donnât aux enfants bègues une gouvernante étrangère : pour s'entretenir avec elle, les enfants auraient à utiliser sa langue et dès lors cesseraient de bégayer. En réalité, l'amélioration de la parole, quand elle se produit, dure aussi longtemps que le bègue n'est pas vraiment familiarisé avec la langue étrangère. Une fois qu'il maîtrise cette langue, le risque est grand qu'il se mette à bégayer quand il l'utilise. Plusieurs hypothèses peuvent être proposées pour expliquer ce fait singulier. Parmi elles, il en est une qui a trait à la neurophysiologie de la parole. Tant que quelqu'un apprend une langue étrangère, il doit rester très attentif et veiller chaque instant à la correction de son articulation, à la justesse de ses choix lexicaux, à l'exactitude de ses constructions syntaxiques. En d'autres termes, il doit constamment diriger son acte de parole, sans pouvoir, comme dans sa langue maternelle, s'en remettre à des automatismes, c'est-à-dire à des habitudes acquises facilitatrices. Alors que le contrôle délibéré de l'acte élocutoire paraît dépendre essentiellement du cortex, les automatismes verbaux semblent dépendre principalement de structures souscorticales. Lorsque ces automatismes se créent dans la langue étrangère par un emploi intensif et prolongé de celle-ci, l'aisance élocutoire s'installe, l'effort d'attention diminue, la fatigue est moindre, car le labeur verbal se trouve réparti entre structures corticales et sous-corticales. Chez le bègue, toutefois, les structures sous-corticales auraient tendance à mal fonctionner et à perturber, par des itérations et des blocages, le bon déroulement de l'acte de parole. Au début de l'apprentissage d'une langue étrangère et tant que ne se sont pas constitués les automatismes verbaux, le cortex assure seul l'expression orale et celle-ci se fait sans achoppement. Mais, à un certain moment, la pratique intensive et prolontée de la langue étrangère fera naître les automatismes, c'est-àdire entraînera l'intervention de structures sous-corticales. Et celles-ci, du fait de leur mauvais fonctionnement, perturberont l'usage de la langue étrangère comme ils perturbent celui de la langue maternelle. Si, par un effort délibéré, le bègue recorticalise l'expression orale, sa fluidité augmente. C'est ce qui se passe lorsque le bègue imite la façon de parler d'un autre locuteur ou joue sur scène un rôle de composition. C'est probablement aussi ce qui a lieu lorsque le bègue ralentit volontairement son débit élocutoire ou étire systématiquement ses voyelles. En fait, toute façon inhabituelle de parler, adoptée délibérément par le bègue, est de nature à diminuer le nombre des achoppements, probablement parce qu'elle réduit la participation des structures sous-corticales.

Cette hypothèse qu'inspire l'étude du bégaiement et qui a trait au rôle respectif des structures corticales et sous-corticales dans l'acquisition et l'utilisation du langage, permet peut-être de résoudre le différend qui oppose les partisans et adversaires de l'ap- 
prentissage précoce de langues étrangères. Les partisans du bilinguisme chez les jeunes enfants font valoir qu'avant l'âge de dix ou douze ans, l'enfant peut apprendre une ou plusieurs langues étrangères bien plus facilement et bien mieux qu'un grand adolescent ou un adulte. Before the age of nine to twelve, a child is a specialist in learning to speak. At that age he can learn two or three languages as easily as one, déclarait Penfield dans l'ouvrage qu'il écrivit avec Roberts (1959:235). Cette idée fut reprise dans l'étude parue en 1965 et dont il a été fait mention au début du présent article: The child is the genius in our society when it comes to acquiring the ... units or the patterns of a language. Mais la thèse que le jeune enfant est avantagé dans l'aprentissage de langues étrangères a été mise en doute par un certain nombre de chercheurs et notamment par Snow et Hoefnagel-Höhle $(1977,1978)$, qui ont montré que des anglophones d'âge préscolaire installés avec leurs parents en Hollande apprenaient moins bien le néerlandais que leurs aînés au cours de la première année passée aux Pays-Bas. On peut toutefois se demander si des observations de ce genre infirment vraiment l'idée d'une période critique pour l'apprentissage des langues. Il se pourrait que la supériorité du jeune enfant n'apparaisse pas d'emblée, mais que ce soit seulement après plusieurs années d'apprentissage que sa connaissance de la langue étrangère se révèle plus intime que celle de l'adulte. De plus, cette meilleure maîtrise pourrait consister principalement en la possibilité de parler la langue étrangère sans fatigue, c'est-à-dire sans diminution des performances verbales même après plusieurs heures, ou plusieurs jours, d'utilisation soutenue de cette langue. Il semble en effet que celui qui acquiert une langue étrangère après la puberté, peut arriver à parler cette langue de façon très satisfaisante, mais à condition de contrôler sans cesse son acte élocutoire, de veiller continuellement à la correction et à l'exactitude de sa production verbale. Ceci demande un gros effort d'attention et de concentration, qui souvent ne peut être maintenu des heures durant : au fil du temps, la production verbale se dégrade. Tout se passe comme si l'adulte, à la différence de l'enfant, ne pouvait plus former ces habitudes facilitatrices qui permettent un usage prolongé de la langue sans fatigue excessive. En d'autres termes, l'adulte ne pourrait plus, comme l'enfant, répartir le labeur verbal entre structures corticales et sous-corticales : seules les structures corticales, consommatrices d'énergie parce que peu automatisées, pourraient intervenir dans le maniement de la langue étrangère. Bien sûr, il s'agit là d'une pure hypothèse. Mais deux constatations au moins semblent la confirmer. La première de ces constatations a trait précisément à l'apprentissage de langues étrangères. Les jeunes enfants paraissent acquérir une langue étrangère sans trop y réfléchir : ils répètent et se répètent volontiers ce qu'ils entendent, de la même manière qu'ils ont appris et continuent d'apprendre leur langue maternelle. Toute différente est l'attitude de l'adulte qui souvent paraît intellectualiser l'approche de la langue étrangère : au lieu de simplement répéter, il réfléchit, analyse, cherche à comprendre le pourquoi, demande qu'on lui explique. La méthode directe ne semble plus lui convenir, peut-être parce que tout se passe maintenant au niveau cortical et que l'acquisition de vrais réflexes verbaux a pour une bonne part cessé d'être possible. Ceci voudrait dire que les structures sous-corticales ne jouent plus qu'un rôle très secondaire lors de l'apprentisage d'une langue par un adulte, alors qu'elles paraissent, chez le jeune enfant, très impliquées dans les activités verbales. On pourrait donc s'attendre à ce qu'une lésion sous-corticale et en particulier thalamique désorganise le langage de façon plus profonde et plus persistante chez l'enfant que chez l'adulte. Or, telle est bien l'impression qu'ont certains cliniciens (voir notamment Lecours 1980 : 597) après avoir constaté que l'aphasie thalamique a généralement un bon pronostic chez l'adulte alors qu'elle peut être définitive chez l'enfant.

Il n'y a bien sûr rien dant tout ceci qui puisse être tenu pour un fait acquis. La neurophysiologie du langage et en particulier celle du bilinguisme est encore très largement 
une terra incognita que seules des études patientes arriveront peu à peu à découvrir. Et l'examen attentif de bilingues atteints de troubles du langage peut contribuer largement, semble-t-il, à cette découverte.

\section{RÉFÉRENCES}

AJAX, E. (1967) : "Dyslexia without Agraphia", Archives of Neurology, 17, pp. 645-652.

ALAJOUANINE, T. (1968) :l'Aphasie et le langage pathologique, Paris, Baillière.

ARONSON, A. (1980) :Clinical Voice Disorders, New York, Decker.

BYCHOWSKI, Z. (1919) : Ueber die Restitution der nach einem Schädelschuss verlorengegangenen Sprachen bei einem Polyglotten ", Monatsschrift für Psychiatrie und Neurologie, 45, pp. 183-201.

DE MENASCE (1973) : Observations d'un disarthrique sur ses moyens de communication ", Journal de Psychologie, 70, pp. 209-220.

GLONING, I. et K. GLONING (1965) : Aphasien bei Polyglotten ", Wiener Zeitschrift für Nervenheilkunde, 22, pp. 362-397.

HINSHELWOOD, J. (1902) : "Four cases of word-blindness", Lancet 1, pp. 358-363.

LEBRUN, Y. et C. LELEUX (1984) : "Central Communication Disorders in Deaf Signers", in NESPOULOUS, J.-L., A.R. LECOURS \& P. PERRON (Eds.), The Biological Foundations of Gestures Motor and Semiotic Aspects, en préparation.

LECOURS, R. (1980) : Corrélations anatomo-cliniques de l'aphasie. La zone du langage", Revue neurologique, 136, pp. 591-608.

MONRAD_KROHN, (1947) : "Dysprosody or Altered "Melody of language", Brain, 70, pp. 405-415.

OJEMANN, G. and H. WHITAKER (1978) : "The Bilingual Brain", Archives of Neurology, 35, pp. 409-412.

PARADIS, M. (1981) :Selective Crossed Aphasia in a Trilingual Patient. Paper read at the Annual Conference on the Neuropsychology of Language, Niagara Falls, March 7, 1981.

PARADIS, M., M.C. GOLDBLUM \& R. ABIDI (1982) : "Alternate Antagonism with Paradoxical Translation Behavior in two Bilingual Aphasic Patients", Brain and Language, 15, pp. 55-69.

PENFIELD, W. (1965) : "Conditioning the Uncommitted Cortex for Language Learning", Brain, 88, pp. 787-798.

PENFIELD, W. \& L. ROBERTS (1959) :Speech and Brain Mechanisms, Princeton (N.Y.), Princeton University Press.

PITRES, A. (1895) :" Étude sur l'aphasie des polyglottes", Revue de médecine, 15, pp. 873-899.

RAPPORT, R., C. TAN \& H. WHITAKER (1983) : "Language Function and Dysfunction among Chinese and English-Speaking Polyglots : Cortical Stimulation, Wada Testing and Clinical Studies", Brain and Language, 18, pp. 342-366. Reproduit en traduction française dans PARADIS, M. et Y. LEBRUN (éd.) (1983) : la Neurolinguistique du bilinguisme, Paris, Larousse.

RIBOT, T. (1881) :les Maladies de la mémoire, Paris, Alcan.

SNOW, C., \& M. HOEFNAGEL-HÖHLE (1977) : "Age Differences in the Pronunciation of Foreign Sounds", Language and Speech, 20, pp. 357-365.

SNOW, C. \& M. HOEFNAGEL-HÖHLE (1978) : "The Critical Period for Language Acquisition : Evidence from Second-Language Learning", Child Development, 49, pp. 1114-1128. 